\title{
Postergar los antibióticos fue aceptado por más de la mitad de los padres de niños con OMA
}

Pragmatic randomized controlled trial of two prescribing strategies for childhood acute otitis media. Little P, Gould C, Williamson I y col. BMJ. 2001; 322:336-42.

\section{Objetivo}

Evaluar una estrategia de manejo con antibiótico (ATB) diferido por 72 hs. contra otra de antibiótico inmediato en niños con otitis media aguda.

\section{Diseño}

Ensayo clínico aleatorizado abierto*

Lugar

42 prácticas generales del sur de Inglaterra.

\section{Pacientes}

315 niños con otalgia aguda y evidencia otoscópica de inflamación (opacidad con eritema o abombamiento o perforación de la membrana) entre 6 meses y 10 años de vida. Se excluyeron los niños en mal estado general.

\section{Intervención}

Se aleatorizó a los niños a recibir amoxicilina según normas nacionales c/8 hs. inmediatamente o a esperar $72 \mathrm{hs}$, antes de reevaluar la situación. Los padres podían utilizarlo antes pero se los alentaba a que esperaran 72 hs. Si en 72 hs. no mejoraban se les administraba el antibiótico.

\section{Medición de resultados principales}

Se utilizaron cuestionarios autoadministrados para evaluar otalgia, estado general, molestia al dormir, nro. de episodios de malestar, escala de dolor (1-10), nro. de cucharadas de paracetamol usadas y temperatura. A su vez, a los padres se les requirió llenar cuestionarios utilizando escalas de Likert* al inicio y al final del tratamiento para evaluar preocupación, satisfacciones y eficacia percibida con respecto al antibiótico, intención de consultar al mismo médico en el futuro y días perdidos en la escuela o el trabajo. El análisis fue por intención de tratar.

\section{Resultados principales}

En el grupo ATB inmediato ( $n=135), 132$ utilizaron ATB. En el grupo ATB diferido $(n=150), 36$ (24\%) utilizaron ATB (29 al segundo día y 11 al tercero). En el seguimiento se perdió el $10 \%$ de los pacientes.

Tabla 1. Antibiótico inmediato contra antibiótico diferido.

Valores expresados en promedio de días de duración (rango).

\begin{tabular}{l|l|l|l|l}
\hline Sintoma & $\begin{array}{l}\text { ATB } \\
\text { inmediato } \\
(\mathbf{n = 1 3 5 )}\end{array}$ & $\begin{array}{l}\text { ATB } \\
\text { difierido } \\
(\mathbf{n = 1 5 0 )}\end{array}$ & \multicolumn{2}{|l}{ Diferencia, (IC 95\%) y p } \\
\hline Otalgia & $2.56(0-10)$ & $3.57(0-11)$ & $-1.10(-0.54$ a -1.48$)$ & $p<0.01$ \\
\hline Molestias al dormir & $1.64(0-8)$ & $2.35(0-11)$ & $-0.72(-0.30 \mathrm{a}-1.13)$ & $p<0.01$ \\
\hline Llanto & $1.54(0-7)$ & $2.23(0-11)$ & $-0.69(-0.31$ a -1.08$)$ & $p<0.01$ \\
\hline $\begin{array}{l}\text { Días de escuela perdidos } \\
1.97(0-8)\end{array}$ & $2.15(0-13)$ & $-0.18(-0.76$ a 0.41$)$ & NS \\
\hline $\begin{array}{l}\text { Nro. Episodios de } \\
\text { malestar por día }\end{array}$ & $0.70(0-4)$ & $0.82(0-7)$ & $-0.12(-0.34$ a 0.11$)$ & NS \\
\hline $\begin{array}{l}\text { Cucharas paracetamol } \\
\text { por día }\end{array}$ & $1.69(0-6)$ & $2.28(0-8)$ & $-0.52(-0.79$ a -0.62$)$ & $p<0.01$ \\
\hline Escala de dolor diario & $2.29(1-5)$ & $2.45(1-6)$ & $-0.16(-0.42$ a 0.11$)$ & NS \\
\hline
\end{tabular}

Valores expresados en número de pacientes (porcentaje)

\begin{tabular}{|l|l|l|l}
\hline & $\begin{array}{l}\text { ATB } \\
\text { inmediato }\end{array}$ & $\begin{array}{l}\text { ATB } \\
\text { diferido }\end{array}$ & $\begin{array}{l}\text { valor } \\
\text { de p p }\end{array}$ \\
\hline Convicción que los ATB son muy eficaces & $100 / 131(76)$ & $64 / 140(46)$ & $p<0.01$ \\
\hline Muy satisfechos con la conducta adoptada & $123(91)$ & $115(77)$ & $\mathrm{p}<0.01$ \\
\hline Intención de consultar a su médico en el futuro & $109 / 132(83)$ & $92 / 147(63)$ & $\mathrm{p}<0.01$ \\
\hline
\end{tabular}

\section{Conclusiones}

La estrategia de ATB inmediato tiene beneficio en la reducción del período sintomático de la enfermedad (cerca de 1 día), que debe ser evaluado contra el aumento de efectos adversos (diarrea) y la perpetuación de la convicción paterna en su eficacia. La conducta de ATB diferida fue aceptada por gran parte de los padres y redujo en un $76 \%$ la utilización de ATB.

\section{Comentario}

La otitis media aguda es una de las infecciones más frecuentes en atención primaria. Aunque la mayoría de los chicos con esta patología recibirán ATB por parte de su médico, su eficacia está discutida.

La evidencia aportada hasta el momento por este y otros estudios sugiere un beneficio moderado en los síntomas (un día menos de duración) a expensas de un aumento en los efectos adversos, mayormente diarrea, sin modificaciones sustanciales en la historia natural de la en-

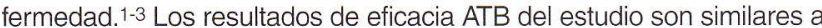
trabajos anteriores con lo que validan en cierta forma los resultados obtenidos en cuanto a satisfacción. Existe controversia acerca de la mayor incidencia de mastoiditis si las OMA no son tratadas con ATB. Un artículo evaluó la incidencia de complicaciones de la OMA sin tratamiento encontrando que cada 4850 pacientes, dos desarrollaron mastoiditis y éstas respondieron bien al tratamiento una vez instaurado. ${ }^{4}$ En los países nórdicos, la estrategia de conducta expectante es implementada desde 1990 sin aumento de las complicaciones. ${ }^{5}$
Hasta la fecha, ningún trabajo había evaluado la aceptabilidad por parte de los padres de una conducta expectante, que es la novedad aportada por este trabajo. Un factor a tener en cuenta es que de los 384 pacientes que inicialmente fueron identificados para el estudio, $69(18 \%)$ no fueron reclutados; siendo una de las principales causas la disconformidad paterna. Esto disminuiría, quizás, el grado de aceptabilidad informado en el artículo. Aún así, si quisiéramos ser conservadores, y repartiéramos esos 69 en los 2 grupos y asumiéramos que todos tomaron ATB desde el primer día, aproximadamente la mitad de los padres habría aceptado una conducta expectante.

Es necesario realizar trabajos a nivel local que nos permitan extrapolar las conclusiones de este trabajo a nuestra realidad tanto cultural como educativa, diferente a la de Inglaterra. Igualmente, ¿habrá llegado el momento de comenzar a replantearnos el tratamiento de la OMA? De ser así, ¿serviría la iniciativa individual o sería mejor una norma de uso generalizado?

\section{Dr. Sebastián García Martí [ Construir Salud ] \\ Dr. Juan Pablo Mouesca [ Clínica Monte Grande ]}

\section{Referencias}

1. Del Mar C Glasziou P, Hayem M. Are antibiotics indicated as initial treatment for children with acute otitis media ? A meta-analysis. BMJ $1997: 314: 1526-9$

2. Glasziou P, Del Mar C, Hayen M et al. Antibiotics for acute otitis media in children (Cochrane Review). En: The Cochrane Library, Issue $4,2000$.

3. Damoiseaux R, Van balen F, Hoes A. Primary care based randomized, double blind trial of amoxicilin versus placebo for acute otitis media in childern aged under 2 years. BMJ 2000;320:350-

4. Van Buchem F, Peeters M, Van't Hof M. Acute otitis media: a new treatment strategy. BMJ 1985, 290:1033-7.

5. Froom J, Culpepper L, Jacobs M. Antimicrobials for acute otitis media? A review fromn the Internatinal Primary Care Network. BMJ 1997; $315: 98-102$. 